\title{
IX. Innenpolitisches Engagement
}

Auch während seiner Tätigkeit auf internationaler Ebene für IIS und FIS blieb Johannes Schauff eng mit dem politischen Leben in der Bundesrepublik verbunden. Wichtige Ansprechpartner waren u. a. Bundeskanzler Adenauer, Staatssekretär Hans Globke, Außenminister Heinrich von Brentano sowie Heinrich Krone, Bruno Heck und Karl Theodor von und zu Guttenberg. Schauffs politischer Rat war gesucht, wo es um die Außen-, besonders um die Entwicklungspolitik ging. So schrieb ihm Bruno Heck, damals Bundesminister für Familie und Jugend, anläßlich der anstehenden Genehmigung eines Ausbildungsprojektes für Brasilien durch das Bundesministerium für wirtschaftliche Zusammenarbeit, diese werde erst erfolgen, „wenn Sie nach Ihrer Orientierung in Brasilien Ihr Votum dazu abgegeben haben" 1 .

Schauff konnte seine immer wieder erklärte Intention, durch seine internationale Tätigkeit auch der Bundesrepublik außenpolitisch zu nützen, sowohl bei den europäischen Nachbarn wie auch in Lateinamerika in vielfacher Hinsicht umsetzen. Parallel zu seinem internationalen Engagement führten jedoch seine umfangreichen Verbindungen und seine Beteiligung an politischen Entscheidungsprozessen in Bonn auch zu einer von ihm ursprünglich keineswegs intendierten Einbindung in die bundesdeutsche Innenpolitik. Dies führte nicht selten zu Spannungen und Überbeanspruchungen.

\section{Die Große Koalition 1966-1969 und die Wahlrechtsfrage}

Im Oktober 1966 brach die CDU/CSU-FDP-Koalition der Regierung Erhard auseinander. Einen Monat später, am 10. November 1966, wurde der amtierende Ministerpräsident von Baden-Württemberg Kurt Georg Kiesinger von der CDU/ CSU-Bundestagsfraktion zum Kanzlerkandidaten bestimmt. Schauffs Anteil am Zustandekommen der daraufhin gebildeten Großen Koalition mit der SPD ist nicht gering einzuschätzen.

Möglichkeit und Notwendigkeit eines Zusammengehens von Sozialdemokraten und Vertretern des politischen Katholizismus waren in konservativ-katholischen Kreisen schon seit langem diskutiert worden; Schauff wie auch Brüning im Exil sowie Vertreter des christlich-konservativen Widerstands gegen die nationalsozialistische Diktatur, die dem ehemaligen Zentrum zuzuordnen sind, sind hier zu nennen ${ }^{2}$. Dahinter stand die Idee von der demokratischen Volkspartei, wie sie auch von Sozialdemokraten vor allem im Londoner Exil entwickelt worden war.

2 Vgl. Morsey, Brünings Einschätzung, S. 267; Becker, Politische Neuordnung, S. 267. 
Ansätze zu einer solchen Großen Koalition nach dem Kriege, wie z.B. in Berlin und im Badischen, waren jedoch an der Zurückhaltung der SPD gescheitert. Schauffs Überzeugung von der Notwendigkeit einer Reform der Parteiendemokratie, die vor allem auf die Erfahrung des Krise der Weimarer Demokratie zurückging, hatten ihn, wie schon angeführt, zum Parteigänger eines mehrheitsbildenden personenbezogenen Wahlrechts werden lassen. In der Bundesrepublik schien sich nun in der zweiten Hälfte der sechziger Jahre angesichts der aktuellen politischen Entwicklung die Möglichkeit zu bieten, im Rahmen einer Großen Koalition ein solches Wahlrecht durchzusetzen, nachdem auch die Sozialdemokraten - offensichtlich aufgrund ähnlicher historischer Erfahrungen - ihre einseitige Fixierung auf das Verhältniswahlrecht aufgegeben hatten. Schauffs Engagement für das Zustandekommen der Großen Koalition war nicht zuletzt von der Erwartung geprägt, eine entsprechende Wahlrechtsreform mit herbeiführen zu können.

Bereits während und nach der langwierigen Regierungsbildung 1961 war innerhalb der CDU aufgrund der politischen Erosion des Bündnisses von Union und FDP die Möglichkeit sowohl einer Großen Koalition wie der Einführung des Mehrheitswahlrechts diskutiert worden; ein maßgeblicher Exponent dieser Perspektiven war der kurz zuvor gewählte Bundespräsident Heinrich Lübke, der zur Unterstützung seiner Kampagne Johannes Schauff aus Brasilien anreisen ließ ${ }^{3}$. Schauffs Einsatz in dieser Frage blieb jedoch ohne Erfolg, weil er in der CDU zu wenig Rückhalt fand. Mit Heinrich Krone habe er eine ganze Nacht darum gerungen, „doch zu Herrn Ollenhauer zu gehen und durch eine alte Freundschaft ein gemeinsames Erbe wirksam zu machen". Doch seien weder Krone noch Heinrich von Brentano von der Auffassung abzubringen gewesen, zunächst durch eine „Ära Erhard“ hindurchzumüssen ${ }^{4}$. Auf christdemokratischer Seite wurde der Vorstoß für eine Große Koalition vor allem von Paul Lücke (CDU) und Karl Theodor von und zu Guttenberg (CSU) unterstützt. Beide Politiker hatten in Zusammenarbeit mit Herbert Wehner bereits in den später gescheiterten Koalitionsverhandlungen Anfang Dezember 1962 eine Koalition aus Sozial- und Christdemokraten möglich zu machen versucht ${ }^{5}$.

Ein weiterer engagierter Vorkämpfer für ein mehrheitsbildendes Wahlrecht war Paul Lücke, Exponent des sozialen Katholizismus und seit 1957 Bundesminister für Wohnungsbau und ab 1961 Minister für Wohnungswesen, Städtebau und Raumordnung. Guttenberg, einer der profiliertesten Außenpolitiker seiner Fraktion, verfocht zusammen mit Lücke, Adenauer und Strauß ein enges Zusammengehen mit Frankreich, wo das Mehrheitswahlrecht bereits Tradition hatte. Guttenberg hatte Wehner im Auswärtigen Ausschuß des Bundestags kennengelernt, im November 1962 war er mit ihm und zusammen mit Paul Lücke zunächst geheimgehaltene Koalitionsverhandlungen eingegangen ${ }^{6}$. Auch nach deren Schei-

3 Interview Erich Kusch mit Johannes Schauff („Die Große Koalition“), in: Welt am Sonntag, 17. 9. 1967; Baring, Machtwechsel, S. $32 \mathrm{ff}$.

4 Schreiben Schauff an Herbert Wehner vom 29. 11. 1965; Schauff an Otto B. Roegele, 4. 1. 1968 (IfZ, NL Schauff, Bd. 25 u. Bd. 36).

5 Vgl. Morsey, Vorbereitung.

6 Vgl. ebenda, S. 463; Wirz, Guttenberg, S. 197 ff. 
tern - Heinrich Krone schrieb am 14. Dezember 1962, daß „für das, was Lücke, Guttenberg und Wehner wollten, die Zeit noch nicht gekommen [sei]" Kontakt zu Wehner, dem „Demokraten, der erfahren hat, daß es nicht um Doktrinen, sondern um Menschen geht ${ }^{\text {" }}$, nicht mehr abgerissen.

Auch Johannes Schauff nahm mit Lücke und Guttenberg Anfang der sechziger Jahre Verbindung auf. Dies geschah im Umfeld der Katholischen Akademie in München, deren Direktor Karl Forster seinerseits zum Kreis derjenigen gehörte, die das Projekt einer Großen Koalition unterstützten. Bereits seit Gründung der Akademie im Jahre 1957 hatte Forster den Gedankenaustausch zwischen katholischen Theologen, Sozialwissenschaftlern und Sozialdemokraten zu fördern gesucht ${ }^{9}$. Auch Guttenberg war um eine Klimaverbesserung zwischen Katholischer Kirche und SPD bemüht ${ }^{10}$, ein Anliegen, das er mit Schauff teilte ${ }^{11}$. Im Rahmen dieser Bemühungen traf Schauff im März 1963 ebenfalls mit Herbert Wehner zusammen, den er noch aus seiner Leipziger Studienzeit kannte ${ }^{12}$.

Zentrales Diskussionsthema der Tagung war die Wahlrechtsfrage, als Referenten fungierten Ferdinand Hermens und Dolf Sternberger, beide engagierte Vertreter eines Mehrheitswahlrechts. Auf dieser Tagung gab sich auch Wehner als Anhänger des Mehrheitswahlrechts zu erkennen ${ }^{13}$. Schauff wertete die Tagung als einen „Erfolg im Sinne einer Großen Koalition“14.

Das Thema Große Koalition blieb auch in den folgenden Jahren auf der Tagesordnung; die treibenden Kräfte gruppierten sich um Bundespräsident Lübke, Guttenberg und Herbert Wehner. Johannes Schauff intensivierte seine persönlichen politischen Kontakte vor allem zu Wehner, mit dem ihn bald ein zunehmend persönlicher und freundschaftlicher werdendes Verhältnis verband ${ }^{15}$. Ein weiterer Schritt auf dem Weg zur Großen Koalition erfolgte während eines Treffens, zu dem Guttenberg (17. Juli 1965) für den 24. Juli 1965 auf sein Weingut „Reichsrat von Buhl“ in der Pfalz eingeladen hatte: Teilnehmer waren neben Guttenberg und Lücke die Sozialdemokraten Wehner und Georg Leber. Zentrales Thema auf die-

7 ACDP, I-028-013/10.

8 Guttenberg, Fußnoten, S. 50.

9 Im Januar 1958 hatte Forster mit seiner Tagung „Christentum und Sozialismus" unter Teilnahme Wehners eine „Sensation ausgelöst“. Vgl. Morsey, Vorbereitung, S. 463; Wirz, Guttenberg, S. 302; zu Forster und der Katholischen Akademie siehe auch Kap. XII, S. $189 \mathrm{ff}$.

$10 \mathrm{Vgl}$. Wirz, Guttenberg, S. $301 \mathrm{ff}$.

11 Siehe unten, S. $158 \mathrm{ff}$.

12 Schauff hatte nach eigener Aussage Wehner in Leipzig kennengelernt, als er dort im Republikanischen Studentenbund aktiv war (persönliche Auskunft gegenüber dem Verfasser).

13 Vgl. Bouvier, Godesberg, S. 199 f.; Wirz, Guttenberg, S. 302 f.

14 Tagebuch Heinrich Krone, 31.3. 1962 (nach einer telefonischen Information Schauffs), ACDP, I-028-072/5.

15 „Schon seit Jahren“, schrieb Schauff später an Otto B. Roegele, „verbindet mich mit Herbert Wehner - ich darf es wohl so nennen - eine Freundschaft, die nicht nur ins Politische, sondern auch ins Religiöse hineinreicht." (Schreiben vom 4.1. 1968, IfZ, NL Schauff, Bd. 36); vgl. auch den Artikel von Hans-Joachim Fischer in der Frankfurter Allgemeinen Zeitung vom 28. 1. 1989 („Karin und Johannes Schauff - kompromißlos und menschenfreundlich“), in dem sich Schauff folgendermaßen äußert: „Herbert Wehner und ich sind durch gemeinsame politische Grundüberzeugungen verbunden.“ 
sem Treffen war wiederum das Mehrheitswahlrecht, daneben die Möglichkeit, eine Notstandsverfassung verabschieden zu müssen ${ }^{16}$. In den folgenden Jahren bis zum Ende der Regierung Erhard am 27. Oktober 1966 hielten Lücke und Guttenberg die Verbindung zu Wehner aufrecht, der seinerseits das Gespräch mit dem Bundespräsidenten suchte.

Am 10. November 1966 wurde Kurt Georg Kiesinger, wie schon angeführt, zum Kandidaten der CDU/CSU für das Bundeskanzleramt gewählt. Kiesinger war jedoch unmittelbar zuvor in der amerikanischen Presse wegen seiner nationalsozialistischen Vergangenheit angegriffen worden ${ }^{17}$. Ein nicht unwesentlicher Anteil an der Richtigstellung solcher Vorwürfe, insbesondere im Ausland und gegenüber den mißtrauisch gewordenen Amerikanern, kam Johannes Schauff zu. Schauff hatte Kiesinger bereits vor 1933 in Berlin kennengelernt, da Kiesinger ebenfalls in dem Kreis um Carl Sonnenschein verkehrte. So konnte er klarstellen, daß Kiesinger ihn nach dem „Röhmputsch“ bzw. der politischen Mordaktion vom 30. Juni 1934 um Unterstützung gebeten hatte, um nach Brasilien zu emigrieren: Kiesinger hatte zu diesem Zeitpunkt als Repetitor bei der juristischen Fakultät der Berliner Universität gearbeitet, und unter seinen Studenten befand sich Volker Koch-Weser, ein Sohn des ehemaligen Reichsministers Erich KochWeser. Anläßlich eines Besuchs bei der Familie Koch-Weser in deren Wohnung, den der Sohn arrangiert hatte, erfuhr Kiesinger das erste Mal von dem Siedlungsprojekt Rolândia. Sein daraufhin gefaßter Plan, nach Brasilien auszuwandern, war jedoch zum einen an finanziellen Problemen, zum andern und wohl in erster Linie an dem Beharrungsvermögen der Familie gescheitert ${ }^{18}$.

Die Wahl Kiesingers zum Kanzlerkandidaten der CDU/CSU war verbunden mit der Option für ein Regierungsbündnis mit der SPD. Dabei war u.a. auch die Einführung eines neuen Wahlrechts wesentlicher Verhandlungsgegenstand. Entsprechende Gesprächskontakte zwischen Kiesinger und dem stellvertretenden SPD-Fraktionsvorsitzenden Herbert Wehner - zu dieser Zeit wegen der schweren Erkrankung Fritz Erlers de facto Fraktionsführer - wurden noch vor den offiziellen Verhandlungen der Parteikommissionen angebahnt. Gerade hierbei spielte wiederum Johannes Schauff eine wichtige Rolle: Über Schauffs Schwager, den Freiburger Rechtsanwalt und Bundestagsabgeordneten Hermann Kopf (CDU),

16 Vgl. Morsey, Vorbereitung, S. 464.

17 Die Washington Post verbreitete am 5.11. 1966 die unzutreffende Information, Kiesinger sei SA-Offizier gewesen und habe als „politischer Kommissar" der Wehrmacht gedient. Tatsächlich war Kiesinger, der der NSDAP seit 1933 angehört hatte, von April 1940 bis Kriegsende als dienstverpflichteter wissenschaftlicher Hilfsarbeiter im Auswärtigen Amt (stellvertretender Leiter der Rundfunkpolitischen Abteilung) beschäftigt. Vgl. Kroegel, Einen Anfang finden!, S. 24 ff. (mit weiterführender Literatur zu Kiesingers NSDAP-Mitgliedschaft).

18 Kiesingers Aussage, er habe Schauff erst „im Herbst 1966“ kennengelernt, „als er mit mir und Herbert Wehner Fühlung aufnahm, um bei der Gründung der Großen Koalition vermittelnd zu helfen" (Kiesinger, Jahre, S. 207), ist nicht zutreffend. Er selbst korrespondierte mit Schauff wegen dessen Bestätigung seiner Emigrationsabsichten (Schreiben Kiesinger an Schauff vom 1.4. 1982, IfZ, NL Schauff, Bd. 34); vgl. auch Interview Erich Kusch mit Schauff („Die Große Koalition“), in: Welt am Sonntag, 17. 9. 1967. 
nahm Kiesinger diskret und indirekt mit Wehner Kontakt auf. Kiesinger, berichtet Schauff, „bat mich, vertraulich den Weg zu Herbert Wehner zu bahnen und als Vermittler mitzuwirken, ehe es zu offiziellen Verhandlungen komme. Von da an fanden am laufenden Band zwei bis drei Wochen lang von mir organisierte Zusammenkünfte im Geheimen statt, meist in der Bonner Vertretung des Landes Baden-Württemberg, gelegentlich auch bei Heinrich Krone."19 Es kam zu rund einem Dutzend solcher vertraulichen Zusammenkünfte, an denen auch der CDUGeneralsekretär und Berater Kiesingers Bruno Heck teilnahm.

Bereits vor seiner Wahl zum Kanzlerkandidaten hatte Kiesinger gegenüber dem Bundespräsidenten seine Option für eine Große Koalition erklärt ${ }^{20}$. Unmittelbar vor Beginn der Koalitionsverhandlungen erzielte Lücke, seit 1965 Innenminister, mit Kiesinger volle Übereinstimmung hinsichtlich der Einführung eines mehrheitsbildenden Wahlrechts. Auch hier assistierte Johannes Schauff als privater Mittelsmann zwischen Kiesinger, Krone, Lücke und Lübke sowie Herbert Wehner. Schauff war auch an Verhandlungen zwischen den Bundesministern Krone und Heck sowie Herbert Wehner über ein Sachprogramm und die personelle $\mathrm{Zu}$ sammensetzung der Großen Koalition beteiligt ${ }^{21}$. Wehner seinerseits verständigte sich vor allem mit Guttenberg, während Heinrich Lübke auf die Sozialdemokraten hinsichtlich einer Großen Koalition einzuwirken versuchte ${ }^{22}$.

Am 25. November 1966 wurde der auf einem Staatsbesuch in Mexiko weilende Bundespräsident von Johannes Schauff und Rainer Barzel darüber informiert, daß das Zustandekommen einer Großen Koalition gesichert sei, und am Tag darauf entwarfen Krone, Heck, Globke und Schauff ein Arbeitsprogramm für die Koalition, ergänzt durch eine Ministerliste ${ }^{23}$. In der Sitzung der Unionsfraktion am 28. November 1966, in der die Entscheidung für die Große Koalition fiel, appellierte Guttenberg in einer Rede leidenschaftlich dafür, bis zu den Bundestagswahlen 1969 Vorkehrungen zur Einführung eines Zweiparteiensystems zu treffen ${ }^{24}$. Am 30. November war die Große Koalition perfekt, wobei vor allem Guttenberg und Lücke von christdemokratischer Seite die Wahlrechtsfrage in die Koalitionsverhandlungen einbrachten. Nach Kiesingers Regierungserklärung vom 13. Dezember 1966 war die Wahlrechtsreform und damit letzten Endes die Begründung eines Zweiparteiensystems nach britischem oder amerikanischem Vorbild vorrangiges Ziel25.

Dieses Ziel verlor jedoch auf beiden Seiten offensichtlich rasch an Bedeutung. Bereits am 30. Januar 1967 äußerte Schauff in einem Brief an Bruno Heck Sorge darüber, „daß man die Frage des Wahlrechts nicht ernsthaft und wirksam und zur

19 Ebenda; vgl. auch Krones rückblickende Feststellung, Schauff habe Kiesinger „geholfen, Kontakt herzustellen" und über ihn seien "gute Wege zu Wehner" gelaufen (ACDP, I-028-073/3); Baring, Machtwechsel, S. 32 ff.; Kroegel, Einen Anfang finden!, S. 34 ff.

20 Tagebuch Berger, 6. 10. 1966, ACDP, I-400-015/1; Morsey, Vorbereitung, S. 470.

21 Tagebuch Berger, 18. 11. 1966, ACDP, I-400-012/1.

22 Ebenda, 15. November 1966, ACDP, I-400-012/1.

23 Ebenda und 25. 11. 1966, ACDP, I-400-012/1.

$24 \mathrm{Vgl}$. Wirz, Guttenberg, S. $459 \mathrm{ff}$.

25 Verhandlungen des Deutschen Bundestags, 5. Wahlperiode, Stenographische Berichte, Bd. 63, 80. Sitzung 13. 12. 1966, S. 3657. 
richtigen Zeit angeht" 26 . Heck äußerte in seinem Antwortschreiben die Skepsis, „daß weder bei den Sozialdemokraten noch bei uns eine klare Mehrheit für das Mehrheitswahlrecht oder auch nur ein mehrheitsbildendes Wahlrecht zu finden ist ... Bei der Unsicherheit darüber, was möglich ist, was erreicht werden kann, ist es nicht ungefährlich für einen Koalitionspartner, sich einseitig hart zu engagieren. Und die Sozialdemokraten behandeln dieses Problem zur Zeit mit bemerkenswerter Behutsamkeit.“27

Diese „Behutsamkeit“ der Sozialdemokraten ließ das Projekt einer grundlegenden Wahlrechtsreform schließlich scheitern. Die frühere politische Fixierung der SPD auf das Verhältniswahlrecht mag dabei durchaus eine Rolle gespielt haben. Folgt man Arnulf Baring, so hatte das Mehrheitswahlrecht für Wehner bereits bei dem Bemühen um eine Große Koalition im Jahre 1962 eine nur taktische Funktion $^{28}$. Aber auch Guttenberg wurde bis zu seinem Tode von der Frage gequält, ob Wehner ihn letzten Endes nicht doch getäuscht habe ${ }^{29}$. Als dann die Große Koalition tatsächlich realisiert worden war, traten alsbald andere innen- und außenpolitische Themen in den Vordergrund. Hinzu kam, daß in Erhebungen bestimmter Wahlforschungs-Institute den Sozialdemokraten schlechte Chancen bei der Einführung des neuen Wahlmodus eingeräumt wurden ${ }^{30}$. Bereits im November 1967 lehnten Wehner und im Januar 1968 die SPD offiziell die Einführung eines neuen Wahlrechts noch im Jahre $1969 \mathrm{ab}$; eine Reform komme frühestens für 1973 in Frage. Auf dem Nürnberger Parteitag der SPD im März 1968 wurde schließlich beschlossen, das Thema erst auf dem nächsten Parteitag $1970 \mathrm{zu}$ behandeln, also nach der nächsten Bundestagswahl. Paul Lücke sah hierin „praktisch ein Begräbnis erster Klasse" für die projektierte Wahlrechtsreform ${ }^{31}$.

Für den amtierenden Innenminister entfiel damit „eine der wichtigsten Voraussetzungen" für die Große Koalition; nicht zuletzt deshalb trat er am 26. März 1968 zurück. Über die Hintergründe dieses Rücktritts und das Scheitern seiner und Schauffs Bemühungen um eine Wahlrechtsreform schrieb Lücke einige Jahre später an Schauff: „Herr Wehner war über jede Einzelheit meiner Absichten informiert. Noch wenige Tage vor dem Weihnachtsfest 1967 fand ein DreierGespräch am Kamin im Bungalow mit Bundeskanzler Kiesinger, Wehner und mir über das Thema [der Wablrechtsreform] statt. Dieses Gespräch währte mehrere Stunden. Ich habe dabei die von der Wahlrechtskommission - Professor Eschenburg - entwickelte und von mir in Gesetzesform gebrachte ,Dreier-WahlkreisKonzeption' vorgetragen, die auch generell die Zustimmung Wehners und Kiesingers gefunden hat. Bei dem dann Anfang Januar 1968 stattgefundenen Koalitionsgespräch unter Vorsitz von Bundeskanzler Kiesinger stellte ich einen völligen

26 IfZ, NL Schauff, Bd. 34.

27 Schreiben vom 9. 2. 1967, IfZ, NL Schauff, Bd. 34.

28 Baring, Machtwechsel, S. $108 \mathrm{ff}$.

29 Wirz, Guttenberg, S. 478.

30 Dies traf zumindest auf das der SPD nahestehende Institut für angewandte Sozialwissenschaft (Infas) zu.

31 Sitzung der CDU/CSU-Bundestagsfraktion, 25. 3. 1968 (Protokolle in ACDP, VIII-001/ 1016-1); zum Scheitern der Wahlrechtsreform während der Großen Koalition vgl. auch Wirz, Guttenberg, S. $476 \mathrm{ff}$. 
Sinneswandel bei Wehner fest, der bei diesem Gespräch plötzlich forderte, man müsse diese Gesetzesvorlage erst einmal näher prüfen usw. Auf dem dann am 28. März in Nürnberg stattgefundenen Parteitag ließ Wehner - nach genau überlegter Strategie - die Wahlrechtsfrage fallen. Für mich bleibt bis zur Stunde hierfür nur die Erklärung, daß Wehner die Wahlrechtsfrage nie gewollt hat, ihm genügte es, mit meiner Hilfe an die Macht gekommen zu sein. Ich hatte schon bei dem Grundsatzgespräch vor Weihnachten darauf hingewiesen, daß, falls die in der Regierungserklärung an hervorragender Stelle festgelegte Wahlrechtsfrage nicht verabschiedet werden würde, ich meinen Rücktritt erklären müsse. Das habe ich dann nach dem Beschluß des SPD-Parteitages vollzogen. Dabei war ich bis zur offiziellen Ankündigung meines Rücktritts der Zustimmung von Bundeskanzler Kiesinger sicher, daß er notfalls die Regierung auflösen und Neuwahlen ausschreiben würde, bevor er zulasse, daß die Wahlrechtsfrage scheitere. Dr. Kiesinger hat sein Wort nicht gehalten. Er hätte damals für unser Land und die CDU diese $\mathrm{Zu}$ sage wahr machen müssen und konnte dabei eines großen Wahlerfolgs der CDU sicher sein." 32

Die Frage nach den Gründen für dieses erneute Scheitern des Projekts einer grundlegenden Wahlrechtsreform, für die sich unter anderen auch Helmut Unkelbach $^{33}$ und Ferdinand Hermens engagiert hatten, ist bis heute nicht gründlich erforscht. Schauff hat später das Scheitern der Großen Koalition vor dem Hintergrund einer ungelösten Wahlrechtsreform dahingehend interpretiert, daß kleineren Parteien wie der FDP - die „lachende Dritte bei diesem System“ -, die „vielleicht sechs, sieben oder acht Prozent der Stimmen“ erreichten, de facto 50 Prozent der Macht eingeräumt würden ${ }^{34}$.

\section{Die Bundespräsidentschaft Heinrich Lübkes}

Das Scheitern der Wahlrechtsreform blieb nicht die einzige Belastung für die Große Koalition, die ja ansonsten innenpolitisch u.a. mit der Verabschiedung der Notstandsgesetze und der Finanzreform durchaus erfolgreich war. Einen zweiten kritischen Punkt bildete die Amtsniederlegung des amtierenden und die Wahl eines neuen Bundespräsidenten. Diese Operation gestaltete sich vor allem in $\mathrm{Hin}$ blick auf das Ende der Präsidentschaft Heinrich Lübkes als äußerst schwierig. Hier war vielen Seiten die Hilfe von Johannes Schauff willkommen.

Schauff hatte schon in der zweiten Hälfte der vierziger Jahre im Zuge der Wie-

32 Brief Paul Lücke an Schauff vom 18. 12. 1974. IfZ, NL Schauff, Bd. 25; Paul Lücke, Bonn, S. $66 \mathrm{ff}$.

33 Helmut Unkelbach (1910-1968), apl. Professor für Politikwissenschaft in Bonn, zahlreiche Arbeiten zu Wahlverfahren und Wahlsystematik; s. Bericht der Arbeitsgruppe für Wahlrechtsfragen der CDU (Unkelbach, August 1965) und die Korr. Schauff-Unkelbach Dezember 1964-November 1965 (IfZ, NL Schauff, Bd. 25).

34 Interview Erich Kusch mit Schauff, November 1974 (Entwurf mit handschriftlichen Ergänzungen Schauffs; eine gekürzte Fassung wurde später im Südwestfunk gesendet; Ms. im Besitz des Verfassers). 
deraufnahme von Kontakten mit Vertretern der ehemaligen Weimarer Siedlungsbewegung auch die Verbindung zu Heinrich Lübke aufgenommen, damals - 1947 - Landwirtschaftsminister in Nordrhein-Westfalen. Mit dem Sauerländer Lübke, in der Weimarer Republik Geschäftsführer des „Reichsverbands landwirtschaftlicher Klein- und Mittelbetriebe“, hatte Schauff das Engagement zur Förderung eines kräftigen christlichen Bauerntums verbunden, das vor allem die damaligen Ostgebiete des Reiches stärken sollte ${ }^{35}$. Die Beziehungen der beiden Familien nahmen rasch freundschaftliche Formen an, die formelle Anrede wurde 1952 durch das vertrauliche „Du“ ersetzt.

Lübke hatte Schauff davon abgeraten, ein Amt in der Bundesregierung oder im diplomatischen Dienst zu übernehmen, das nur seine politische Bewegungsfreiheit einschränken würde ${ }^{36}$. Dagegen unterstützte er Schauffs Aktivitäten in der ICMC, darunter das weiter oben angeführte Siedlungsprojekt in Südfrankreich. In den Kontakten und Begegnungen mit Lübke und mit anderen ehemaligen Siedlungspolitikern zeigte sich für Schauff, daß es in diesem Kreis eine grundlegend skeptische Haltung gegenüber der neuen Bonner Regierung gab, von der man glaubte, daß sie die - zum Teil emigrierten - alten Weimarer Eliten und insbesondere die gescheiterten Agrarpolitiker ausgrenzen wolle. In einem Schreiben von Karl Fütterer an Schauff vom 22. September 1949, das offenbar eine in diesen Kreisen weitverbreitete Stimmung wiedergibt, heißt es: „Wir sind froh, daß sich Lübke nicht in die Bonner Gesellschaft begeben hat. Daß man Niklas ${ }^{37}$ genommen hatte, nachdem selbst Karl Müller ${ }^{38}$ und Schlange-Schöningen verzichtet hatten, sagt ja über den Verein mehr als genug aus. Es wird Sie interessieren, daß Adenauer den Vertretern der Bauernverbände, darunter Hermes, erklärt hat, er könne ihren Wunsch, Lübke in das Kabinett aufzunehmen, nicht erfüllen, nachdem dieser ein solches Bodenreformgesetz gemacht habe." 39

Adenauer selbst war kein Freund von „bodenreformerischen Experimenten“. Er sah in Lübke, der 1946 an der Wiederbelebung des Gedankens der „inneren Kolonisation" beteiligt war und sich für eine gezielte Boden- und Siedlungspolitik einsetzte, die die sozialen Belange insbesondere der Vertriebenen und Flüchtlinge berücksichtigte, einen zu weit vorpreschenden „Bodenreformer“, den er aus der CDU-Politik herauszuhalten versuchte ${ }^{40}$. Lübke machte in Bonn gleichwohl seinen politischen Weg: 1959 wurde er als Nachfolger von Theodor Heuss Bundespräsident ${ }^{41}$. Schauff gehörte alsbald zum engeren Beraterkreis des neu gewählten

${ }^{35}$ Vgl. Morsey, Heinrich Lübke, S. $68 \mathrm{ff}$.

${ }^{36}$ Brief Heinrich Lübke an Schauff vom 1. 4. 1950 (IfZ, NL Schauff, Bd. 5).

37 Wilhelm Niklas/CSU, 1949-1953 Minister für Landwirtschaft und Ernährung im 1. Kabinett Adenauer.

$38 \mathrm{Karl}$ Müller/CDU, war in der 1. Zivilregierung der Nordrhein-Provinz 1945/46 Abteilungsleiter für Ernährung und Landwirtschaft, 1946-1950 MdL Nordrhein-Westfalen.

39 IfZ, NL Schauff, Bd. 33; zu Lübkes Bodenreformplänen nach 1945, der Genesis des Bodenreformgesetzes und seiner Politik als Landwirtschaftsminister in Nordrhein-Westfalen (1947-1952) vgl. Morsey, Heinrich Lübke, S. $145 \mathrm{ff}$. und $171 \mathrm{ff}$.

40 Morsey, Heinrich Lübke, S. $146 \mathrm{ff}$.

${ }^{41}$ Hierzu umfassend und auf dem neusten Forschungsstand die Lübke-Biographie von Morsey. 
Bundespräsidenten. Unterstützt wurde er dabei von Heinrich Krone, der sich darum sorgte, daß dem "westfälischen Dickkopf“ Lübke ein Mann zur Seite stehe, auf den dieser höre ${ }^{42}$.

Neben der gemeinsamen beruflichen Erfahrung waren es vor allem Schauffs aktuelle internationale Erfahrungen und Beziehungen, die Lübke manchen Rat annehmen ließen. Besonderen Stellenwert besaß in diesem Zusammenhang, wie schon angeführt, das Projekt einer künftigen Großen Koalition. Schauff vermittelte auch Herbert Wehner den Kontakt zur Villa Hammerschmidt, deren Hausherr seinerseits den sozialdemokratischen Politiker zu schätzen wußte. Soweit Schauffs internationale und familiäre Verpflichtungen in Europa und Lateinamerika dies zuließen, kam er häufig mit Lübke zusammen ${ }^{43}$. Er beriet den Bundespräsidenten aber nicht nur, sondern bemühte sich auch um sein positives Image in der Öffentlichkeit ${ }^{44}$. Im internationalen bzw. außenpolitischen Bereich konnte er eigene Erfahrungen nicht nur theoretisch vermitteln, sondern war vielfach auch vor Ort präsent, z.B. als Lübke vom 20. bis 23. Juni 1961 seinen ersten Staatsbesuch in Frankreich absolvierte ${ }^{45}$. Die Wiederwahl Lübkes am 1. Juli 1964 mit der Mehrheit der sozialdemokratischen Wahlmänner ${ }^{46}$ markierte schließlich eine wichtige Etappe auf dem Weg in die Große Koalition ${ }^{47}$.

Noch einmal - während der zweiten Amtszeit Lübkes - spielte Schauff eine wichtige vermittelnde Rolle, als es um die Neubesetzung des Staatssekretärspostens im Bundespräsidialamt ging. Schon Ende 1960 hatte Schauff sich in die Suche nach einem neuen Chef des Bundespräsidialamtes eingeschaltet ${ }^{48}$. Als Nachfolger von Staatssekretär Hans von Herwarth wurde im Frühjahr 1965 vom Auswärtigen Amt der amtierende Botschafter in Den Haag, Hans Berger, vorgeschlagen. Berger, Schauff aus der Zentrums-Jugend bekannt, bat diesen um Unterstützung bei seiner Kandidatur. Schauff erfüllte diesen Wunsch gerne, warnte Berger aber zugleich vor dem „sehr schwierigen Job“, zu dem man Freunden eigentlich ungern zurate ${ }^{49}$. Berger vermochte sich gleichwohl in dem neuen Amt gut einzuarbeiten ${ }^{50}$.

42 Dem neugewählten Bundespräsidenten fehle ein Globke, meint Krone in seinem Tagebucheintrag vom 7. 2. 1960 (Krone, Tagebücher, S. 404). Hintergrund dieser Sorge war die zunehmende Einflußnahme der Präsidentengattin Wilhelmine Lübke. Vgl. auch Morsey, Heinrich Lübke, S. $302 \mathrm{ff}$.

$43 \mathrm{Vgl}$. auch Herwarth, Adenauer, S. 315.

44 Vgl. die auf Anregung von Schauff von Oskar Simmel SJ, dem Chefredakteur der Stimmen der Zeit, am 4. 7. 1960 brieflich ausgesprochene Wertung der Arbeit des Bundespräsidenten als sachlich und staatspolitisch von „höchst bedeutende(r) Art“ (Morsey, Heinrich Lübke, S. 312).

45 Heinrich Krone vermerkt in seinem Tagebuch am 24. 6. 1961, daß Schauff mit dem Auftreten Lübkes in Paris „sehr zufrieden“" gewesen sei (Krone, Tagebücher, S. 510).

46 Im Vorfeld der Wahl hatte der persönliche Referent Lübkes, Hermann Sehrbrock, Schauff die Sorge übermittelt, daß die Unionsfraktion Lübkes Kandidatur nicht nachdrücklich genug unterstütze (Brief vom 19. 12. 1963, IfZ, NL Schauff, Bd. 37).

47 Baring, Machtwechsel, S. $34 \mathrm{ff}$.

48 Schauff an Krone, 29. 12. 1960 (ACDP, I-028-014/1); Morsey, Heinrich Lübke, S. 303.

49 Korrespondenz Schauff-Berger in IfZ, NL Schauff, Bd. 1.

50 Berger schrieb am 5. 8. 1965 an Schauff: „Hier lassen sich die Dinge recht erfreulich an. Ich 
"Lübkes beschwerliches Ende“ - so Arnulf Baring - war vor allem auf einen altersbedingten zerebralen Kräfteverfall zurückzuführen, den der Bundespräsident selbst nicht mehr kritisch zu reflektieren vermochte ${ }^{51}$. Der Druck hinsichtlich einer Ablösung wurde durch Berichte in der Presse über Pannen und Entgleisungen verstärkt, die ab 1963 in die Öffentlichkeit kamen. Das Amt des Bundespräsidenten wurde aber vor allem dadurch beschädigt, daß von seiten der DDR eine Desinformationskampagne lanciert wurde, in der Lübke die Beteiligung bei der Planung von Arbeitsunterkünften vorgeworfen wurde, die für KZ-Häftlinge bestimmt gewesen seien. Diese Vorwürfe wurden auch von der Presse in der Bundesrepublik hochgespielt ${ }^{52}$.

Im Rahmen dieser Kampagne wurde auch das Strafverfahren gegen Lübke und seine Untersuchungshaft in den Jahren 1934/35 "ausgegraben“, die vor dem Hintergrund der politischen Verfolgung der damaligen Repräsentanten der Land- und Siedlungsbewegung und des Zentrums zu sehen sind. Nun drohte dieser Tatbestand der Verfolgung eine rein strafrechtliche Dimension zu erhalten und damit zur weiteren Demontage der Person des Bundespräsidenten beizutragen. In dieser Lage wurde Johannes Schauff von Herbert Wehner darum gebeten, bei dem in dieser Angelegenheit aktiven Chefredakteur des Stern, Henri Nannen, persönlich zu intervenieren. Schauff empfahl Staatssekretär Berger auch, die Öffentlichkeit über die „damaligen Zeitverhältnisse“, d.h. über den Tatbestand der politischen Verfolgung der Repräsentanten der Siedlungsbewegung, zu informieren ${ }^{53}$.

In einem Brief an Wehner präzisierte Schauff den gesamten Vorgang und das Verhalten Lübkes, wie er es realistischerweise sah: „Mit der Angelegenheit bin ich schon seit Jahren befaßt und ich habe mich gleich bei seinem St[aatssekretär] G. ${ }^{54}$ $\mathrm{B}[$ erger] nach dem Stand der Dinge erkundigt und meine Bereitschaft zu einer wohlkoordinierten - Aktion erklärt. Er war der Ansicht, daß die aktuelle Gefahr eingedämmt sei, auch dank der Mithilfe des Justizministers, und daß man das

blicke nunmehr auf eine zweimonatige und mich sehr befriedigende Tätigkeit zurück. Sie ist in jedem Falle sehr interessant und die Zusammenarbeit mit dem Herrn Bundespräsidenten ist in der Tat von gegenseitigem Vertrauen getragen." (IfZ, NL Schauff, Bd. 1).

51 Baring, Machtwechsel, S. 37 ff.; Morsey, Heinrich Lübke, S. 483.

52 Ebenda, S. $505 \mathrm{ff}$.

53 "Ich weiß nicht", schrieb Wehner am 1. 3. 1967 an Schauff, „ob Ihnen klar ist, wie H[einrich] L[übke] fortgesetzt beschossen wird ... Der Stoß, den Herr Nannen vor hat, soll ihn wahrscheinlich zu einer Zeit und unter Umständen treffen, die sowieso verwirrend für viele Leute sind. Das Ganze würde mit ,Akten' garniert sein, die den Einruck der Seriosität der Behauptungen hervorrufen sollten ... Weil ich Sie als einen Mann schätze, der weiß, wie Deutschland seinerzeit in sein Unglück gezogen wurde, bitte ich Sie herzlich, sich einmal ganz und gar in die Rolle und Aufgabe eines Mannes zu versetzen, dessen Wissen, Erfahrung und Mut durch keinen anderen zu ersetzen sind, wenn es sich darum handeln sollte, in der Sache H.L. deutlich zu machen, warum H.L. und aufgrund welcher Interessen er damals zur Strecke gebracht werden sollte." In einem späteren Brief Wehners vom 10. 7. 1968 an Schauff heißt es, es sei er, Schauff, gewesen, der Anfang 1967 eine groß angelegte Kampagne gegen Lübke habe verhindern helfen können (IfZ, NL Schauff, Bd. 25 und Bd. 38; das Schreiben Schauff an Berger vom 6. 3. 1967, ACDP, I-400-031/2). Vgl. auch Morsey, Heinrich Lübke, S. $526 \mathrm{ff}$., 548.

54 Gemeint ist $H$. (Hans) Berger. 
Ergebnis des Gutachtens anhand der umfangreichen Gerichtsakten von Staatssekretär Bülow abwarten solle ${ }^{55}$... Jedenfalls habe ich eine Anregung zu einer Besprechung alter Freunde aus der Zeit, die auch den Sachverhalt kennen, aufgegriffen und für Mitte April vorgeschlagen (u.a. Heinrich Krone, Franz Schürholz ${ }^{56}$, Minister Josef Franken). Dabei wird im Vordergrund stehen, wie man einen Fall Ebert II abwenden, denselben bei der gesundheitlich-biologischen Situation von $\mathrm{H}[$ einrich] $\mathrm{L}[\ddot{u} b k e]$ überhaupt abwenden kann, was zu geschehen hat und wie man vorbeugt, wenn eine ähnliche Situation wie bei Segni in Italien eintritt $t^{57}$. Ich glaube, ich deutete Ihnen schon an, daß ich mir aufgrund der Beobachtungen des häufigen persönlichen Zusammenseins in den letzten Monaten wachsende Sorgen mache, ob und wie die zweieinhalb Jahre ohne Zwischenfälle und Gefährdung für Person und Amt - zu Ende gebracht werden können. Dies selbst dann, wenn ich die Loyalität seiner Mitarbeiter und Berater sehr hoch veranschlage. Dazu kommt folgendes: 1933 wurde der Kampf gegen die Träger der Agrarreform und Siedlungspolitik von den Junkern und Deutschnationalen mit ihrem Anhang geführt, die auch heute nicht ohne Einfluß sind und ihr damaliges Verhalten ungern zugeben wollen. Entgegen meinem Rat hat H. L. bei seinem Amtsantritt veranlaßt, daß über diesen Hintergrund seines Lebens nicht gesprochen werden sollte. (Auch hatte ich die notwendigen Schritte unternommen, daß das Institut für Zeitgeschichte die Osthilfe- und Agrarpolitik vor und nach 1933 untersucht ${ }^{58}$. H. L. winkte $\mathrm{ab}$ und auch der von mir vorgeschlagene Historiker Hans Buchheim kam mit seinen Auffassungen nicht durch). Damals waren der Landbund und der dahinterstehende Adel die Gegner des sozialdemokratischen Ministerpräsidenten Braun, von Otto Klepper und Staatssekretär Krüger; dazu kamen Katholiken, ,die

55 Das erwähnte Gutachten über das Verfahren gegen Lübke 1934/35 wurde von Arthur Bülow (bis 1966 Justiz-Staatssekretär) am 27.6. 1967 vorgelegt. Darin wurde dargelegt, daß Lübke aus politischen Gründen und zu Unrecht verfolgt worden sei. Zu dem Vorgang vgl. Morsey, Heinrich Lübke, S. $528 \mathrm{ff}$.

56 Dr. Franz Schürholz, Inhaber einer Kokosweberei, gehörte zum engeren Freundeskreis von Heinrich Lübke; er hatte Lübke bereits während dessen Haftzeit 1934-1935 unterstützt (Morsey, Heinrich Lübke, S. 102 f.). Schürholz hatte am 7. Juni 1968 ebenfalls - vergeblich - bei Heinrich Lübke wegen dessen vorzeitigen Amtsverzichts interveniert (Tagebuch Krone, ACDP I-028-076/2); in einem Schreiben vom 11.7. 1968 an Schauff, dem Schürholz freundschaftlich verbunden war, bat er Schauff um eine eigene Intervention, da Schauff von jeher das "taktische Vorgehen“ gut beherrscht habe. Er beklagte zugleich den Immobilismus der „vorrangig interessierten Partei“, d.h. der CDU (IfZ, NL Schauff, Bd. 25; die weitere Korrrspondenz Schauff-Schürholz, betr. u.a. dessen Engagement für eine deutsch-israelische Aussöhnung, IfZ, NL Schauff, Bd. 7).

57 Schauff spielt hier wohl auf die Kampagne der politischen Rechten an, die Reichspräsident Friedrich Ebert wegen seiner Haltung bei dem Munitionsarbeiterstreik während des Krieges des Landesverrats beschuldigten (vgl. Witt, Friedrich Ebert, S. 169f.); Ebert verstarb am 28. 2. 1925 unerwartet an den Folgen einer Blinddarm- und Bauchfellentzündung. Der italienische Staatspräsident Antonio Segni hatte während seiner Amtszeit am 7. 8.1964 einen Schlaganfall erlitten, dessen Folgen ihn am 7. 12. 1964 zum Rücktritt zwangen.

58 Korrespondenz Hans und Karl Buchheim-Johannes Schauff in IfZ, NL Schauff, Bd. 1. Zur Beziehung Schauffs zum Institut für Zeitgeschichte und seinen zeithistorischen Forschungsinteressen siehe S. 196; zu Karl Buchheims Mitarbeit im Institut für Zeitgeschichte 1950-1952 vgl. Buchheim, Lebensgeschichte, S. $254 \mathrm{ff}$. 
Rom vor die Tore Pommerns und Ostpreußens brachten' und so vogelfrei wie die Linke selbst waren. Wird H. L. heute mit seinen Nerven der Zermürbungstaktik dieser Leute gewachsen sein und wird er nicht selbst unnötige Vorwände liefern? Sie, lieber Herr Wehner, der Sie ein Leben voll von Verleumdung und Verfolgung durchgestanden haben, werden eher ermessen können, was dazu nötig ist." 59

Schauff riet zum Abwarten auch gegenüber der Presse und zu konzertierter Aktion der Freunde. Doch war angesichts der Wirkungen der KZ-Kampagne aus der DDR und in der westdeutschen Presse sowie dem zunehmend problematischen Erscheinungsbild des Bundespräsidenten in der Öffentlichkeit Lübkes Ausscheiden aus dem Amt inzwischen auch von der Regierungskoalition forciert worden.

Nachdem im Frühjahr 1968 in der Öffentlichkeit Spekulationen über einen bevorstehenden Rücktritt Lübkes laut geworden waren, unternahmen christdemokratische Spitzenpolitiker bis hin zu Bundeskanzler Kiesinger bei Lübke entsprechende Vorstöße, die aber offenbar nicht deutlich genug waren bzw. nichts fruchteten ${ }^{60}$. Im Einvernehmen mit Kiesinger und Herbert Wehner sprach daraufhin auch Johannes Schauff am 2. Juli 1968 bei seinem alten Freund und politischen Weggefährten vor. Auch diesem ersten Versuch war ein nur partieller Erfolg beschieden.

Nach einer vorbereitenden Absprache auf Schloß Guttenberg, an der Bundeskanzler Kiesinger und Minister Georg Leber teilnahmen, unternahm Schauff einen weiteren Versuch, bei dem ihm von sozialdemokratischer Seite Außenminister Willy Brandt und Herbert Wehner Rückendeckung versprachen. Schauff erbat sich für die anstehende Überzeugungsarbeit von beiden Politikern flankierende Schreiben, eine Bitte, der jedoch nur Wehner nachkam. Wehners Argumentationshilfe war jedoch besonders wertvoll, nicht zuletzt auch deshalb, weil Lübke in Wehner den Mann sah, dem er vor allem seine Wiederwahl verdankte ${ }^{61}$.

In dem Schreiben, das Schauff bei seinem zweiten Treffen am 16. Juli 1968 Lübke zu lesen gab, äußert Wehner seinen Respekt vor der „moralischen Integrität des Bundespräsidenten“, den er aus politischer Überzeugung und mit allen Kräften immer verteidigt habe. Doch sollte es angesichts des körperlichen $\mathrm{Zu}$ stands des Bundespräsidenten und seiner zunehmenden Schwierigkeiten, die Bürde des Amtes so zu tragen, daß es nicht beschädigt werde, möglich sein, „daß ein ehrbarer Freund des Präsidenten, wie Sie [Schauff] es sind“, ihm nahelegt, „dieser unleugbaren Tatsache“ Rechnung zu tragen. Wehner erinnerte daran, daß er den Bundespräsidenten immer gegen Hetze verteidigt habe, daß jedoch „die schrecklichste Gefahr für das Amt und den guten Namen des Herrn Bundespräsi-

59 Schreiben Schauff an Wehner vom 6. 3. 1967. Einen Monat früher, am 13.2. 1967, hatte Lübke an Schauff geschrieben: „Meine Mitwirkung an der Bildung der neuen Bundesregierung haben mir gewisse Kreise anscheinend sehr übel genommen; denn es gibt in letzter Zeit gehässige Stimmen aus diesem Lager gegen mich.“ (IfZ, NL Schauff, Bd. 38).

60 Morsey, Heinrich Lübke, S. 564 ff.

61 Schauff, Vertrauliche Bemerkung zu dem Brief von Herbert Wehner vom 10.7. 1968 (10. 12. 1977), IfZ, NL Schauff, Bd. 25. 
denten die Lächerlichkeit ist, die sich nunmehr ausbreitet ... Ich möchte, daß das Amt, in das ich Dr. Heinrich Lübke mit gewählt habe, nicht Schaden leide. Ich möchte, daß er selbst nicht Schaden nehme ... Ich möchte, daß ... Lübke als ein Ehrenmann, ein solider Präsident und als ein aufrechter Mensch geschätzt wird und in die Geschichte eingeht."62

Auch dieses zweite Zusammentreffen Schauffs mit Lübke, das zwei Stunden dauerte und nach Schauffs Aussage in einer menschlich angenehmen Atmosphäre verlaufen sei, führte nicht zu dem gewünschten Erfolg63. Schauff glaubte dennoch, daß seine Intervention und vor allem Wehners Brief - sozusagen mit Verzögerungseffekt - nicht ohne Wirkung geblieben seien ${ }^{64}$. Er versuchte es deshalb noch einmal mit einer konzertierten Aktion, da er glaubte, daß Lübke, wenn er die volle Tragweite seiner Intervention erfasse, versuchen werde, Freunde zu mobilisieren, die dann seiner, Schauffs, Meinung möglicherweise beipflichteten. Auf Anraten von Frau Wehner versuchte Schauff auch, den der Familie Lübke freundschaftlich verbundenen vatikanischen Nuntius in Tokio, Bruno Wüstenberg, einzuschalten, der sich von einem solchen Schritt allerdings wenig versprach ${ }^{65}$.

Am 17. Juli 1968 suchte Schauff Bundeskanzler Kiesinger auf, um ihn über seine Mission und vor allem die Hilfestellung durch Wehner ins Bild zu setzen; mit Wehner selbst beriet er sich noch einmal eingehend bei einem familiären $\mathrm{Be}$ such in dessen Feriendomizil in Schweden.

Schauff beklagte sich später vor allem über die Unschlüssigkeit bzw. das mangelnde Durchsetzungsvermögen Kiesingers in der Angelegenheit ${ }^{66}$. Der schwierige Prozeß der Ablösung Lübkes wies freilich durchaus weitere Facetten auf vor allem der Eigensinn des westfälischen Bundespräsidenten, der an seine ungebrochene Popularität sogar bei Jugendlichen glaubte ${ }^{67}$, verhinderte die Lösung

62 Wehner an Schauff, 10. 7. 1968 (IfZ, NL Schauff, Bd. 25).

63 Vgl. Brief Schauff an Kurt Birrenbach vom 29. 7. 1968 (IfZ, NL Schauff, Bd. 25); in einem Brief an Guttenberg vom 30. 7. 1968 berichtet Schauff über die Erfolglosigkeit seines Bemühens, „obwohl er [Lübke] am Tage vorher Herrn Birrenbach wie auch Heinrich Krone zu sich gerufen hatte, um den zu erwartenden Vorstoß (er hatte gemeint, ich käme im Auftrag von Barzel) abzuwehren ... Ich mußte mir die vom Bundeskanzler in Ihrer Gegenwart erteilten Vollmachten voll ausnützen und einen offiziellen Schritt der Parteiführer ankündigen." Auf die Vorlage von Herbert Wehners Brief habe er nur mit Apathie reagiert. „Ich bezweifele es, ob er die Tragweite meiner Intervention überhaupt erfassen konnte ... Im Übrigen war er freundlich in seinem Verhalten wie in alten Zeiten, über die auch viel gesprochen wurde. Den Zentralpunkt, daß sich jetzt seine Freunde um ihn Sorge machen und ihn bewegen wollen, seinen Jahren Rechnung zu tragen, versteht er nicht, er wittert überall politische Manöver ..." (IfZ, NL Schauff, Bd. 33 sowie NL Guttenberg, Nr. 42).

64 In einem Brief an Herbert Wehner vom 23. 7. 1968 berichtet Schauff, daß nach dem Urteil sachverständiger Ärzte „meine fast drastisch zu nennende Einwirkung jedoch später ihm in der vollen Bedeutung aufgeht." Vgl. auch Schauffs Brief an Heinrich Krone vom 24. Juli 1968 und an Staatssekretär Hans Berger vom 25. 7. 1968 (IfZ, NL Schauff, Bd. 25).

65 Schauff an Herbert Wehner, 23. 7. 1969 (IfZ, NL Schauff, Bd. 25).

66 Schauff, Autobiographische Notizen/Fragmente. Die „Entscheidungsschwäche“ Kiesingers wurde offenbar auch in seiner eigenen Regierungsmannschaft beklagt, so u.a. von Regierungssprecher Conrad Ahlers (vgl. Kroegel, Einen Anfang finden!, S. 276).

67 Tagebuch Berger, 17. 7. 1968 (ACDP, I-400-012/1). 
des vorzeitigen Rücktritts. Eines der Argumente, die Lübke zu einem vorzeitigen Amtsverzicht bewegen sollten, lautete, daß zwischen der anstehenden Wahl des Bundespräsidenten im Sommer und der Wahl des Bundestags im Herbst 1969 ein größerer zeitlicher Abstand liegen müsse, um erstere nicht in den Sog des Wahlkampfes zum Bundestag geraten zu lassen. Aber auch diese „staatspolitische“ Begründung sowie weitere persönliche Interventionen anderer Politiker68 vermochten die Dinge erst nach weiterem Drängen in Bewegung zu bringen: Am 19. September 1968 teilte Lübke dem Bundeskanzler seinen Entschluß mit, am 2. Juli 1969 zurücktreten zu wollen. Am 14. Oktober schließlich, anläßlich eines Empfangs in der Villa Hammerschmidt aus Anlaß seines 74. Geburtstags, gab der Bundespräsident dann den definitiven Rücktrittstermin bekannt: am Vorabend des 1. Juli 1969, zehn Jahre nach seiner ersten Wahl.

Johannes Schauff hatte - vor allem im Zusammenspiel mit Herbert Wehner einen wesentlichen Anteil daran, daß das Problem der Präsidentschaft Heinrich Lübkes in dessen letzten Amtsjahren politisch und persönlich einvernehmlich gelöst wurde. Als sich Lübke nach seinem Rücktritt darum bemühte, als Berater der FAO nach Rom zu gehen, um auf diese Weise sein Engagement für die Dritte Welt weiterzuführen, informierte und beriet er sich mit Johannes und Karin Schauff69. Auch als die nicht leichte Amtszeit von Staatssekretär Berger zu Ende ging, für dessen Berufung Schauff sich seinerzeit eingesetzt hatte, wurde Schauff wiederum aktiv. Im Einvernehmen mit Krone wie mit Herbert Wehner und Außenminister Willy Brandt vermittelte er die Berufung Bergers als Nachfolger des verstorbenen Dieter Sattler zum Botschafter beim Vatikan ${ }^{70}$.

\section{Mittler zwischen SPD und Katholischer Kirche}

Schauffs Engagement galt nicht nur der Einbindung der Sozialdemokraten in die Regierung, er war bei dieser Gelegenheit auch bemüht, das Verhältnis der katholischen Kirche zur SPD zu verbessern, die ihrerseits immer noch nicht den Bannstrahl der Enzyklika "Quadragesimo anno" überwunden zu haben schien; dies zeigte sich besonders in ihrer nach wie vor fehlenden Akzeptanz bei der katholischen Wählerschaft.

Bereits in der Weimarer Republik war Schauff über den Kreis um Carl Sonnenschein und dann über die „Religiösen Sozialisten“ mit dem „Arbeiterproblem" vertraut gemacht worden und dabei auch mit Sozialdemokraten zusammengetroffen, mit denen ein "christlicher Diskurs“ durchaus möglich war. Nun schien ihm nach der gesellschaftspolitischen Öffnung der SPD, wie sie sich im Godesberger

68 Bischof Hermann Kunst und Ministerpräsident Helmut Lemke (Schleswig-Holstein) am 26.7., der SPD-Fraktionsvorsitzende Helmut Schmidt am 29. und Bundesminister Bruno Heck am 30. 7. 1968 (Morsey, Heinrich Lübke, S. 566 f.).

69 Schauff, Autobiographische Notizen/Fragmente.; Heinrich Lübke an Johannes und Karin Schauff, 30. 10. 1968 (IfZ, NL Schauff, Bd. 35).

70 Berger erhielt im Mai 1969 das Agrément als Botschafter beim Heiligen Stuhl. Vgl. Korrespondenz Schauff- Berger, IfZ, NL Schauff, Bd. 1; Morsey, Heinrich Lübke, S. $369 \mathrm{f}$. 
Programm manifestiert hatte, mit dem Zweiten Vatikanischen Konzil und der Etablierung der Koalition von Christ- und Sozialdemokraten die Möglichkeit einer Neuorientierung im Verhältnis von SPD und Katholischer Kirche gegeben zu sein; dieser Weg wurde auch von Guttenberg und Karl Forster engagiert verfolgt.

In die schon seit Mitte der fünfziger Jahre von sozialdemokratischer Seite unternommenen Bemühungen, das Verhältnis zum Vatikan und zur katholischen Kirche zu entkrampfen, waren Karl Forster und die von ihm geleitete Katholische Akademie ${ }^{71}$ sowie Gustav Gundlach SJ und der katholische Hochschullehrer Gustav E. Kafka eingebunden; die Exponenten auf sozialdemokratischer Seite waren vor allem Adolf Arndt, Waldemar von Knoeringen und Carlo Schmid. Diese Bemühungen waren bislang von der Amtskirche allerdings nicht angenommen worden ${ }^{72}$. Doch waren, wie sich zeigen sollte, das veränderte politische Klima in der Bundesrepublik Deutschland und insbesondere das Votum der SPD für eine Wiederwahl Lübkes 1964 in vatikanischen Kreisen aufmerksam registriert wor$\operatorname{den}^{73}$.

Schauff war zusammen mit Bruno Wüstenberg wesentlich daran beteiligt, daß im gleichen Jahr 1964 in Rom die erste Begegnung deutscher Sozialdemokraten mit dem damaligen Papst Paul VI. zustande kam ${ }^{74}$. Zur Vorbereitung dieses Besuchs im Vatikan, der am 5. März 1964 stattfand, waren die Sozialdemokraten im Vorfeld auch selbst tätig geworden. Entsprechende Kontakte waren im Oktober 1963 im Einvernehmen mit Herbert Wehner von dem SPD-MdB Ernst Paul über einen Bruder des Papstes, Senator Ludovico Montini, hergestellt worden und wurden später von dem Abteilungsleiter im SPD-Parteivorstand Alexander Kohn-Brandenburg weitergeführt ${ }^{75}$. Auf ein informelles Gesuch an das vatikanische Staatssekretariat hin war am 29. Februar 1964 eine Privataudienz beim Papst für vier Personen genehmigt worden.

Der SPD-Besuchsdelegation gehörten Fritz Erler, Waldemar von Knoeringen, Ernst Paul und Peter Nellen ${ }^{76}$ an. Zu Beginn der Audienz, bei der Bruno Wüstenberg als Dolmetscher fungierte, fand ein kurzes, gesondertes Gespräch des Papstes mit Fritz Erler statt, das die geistige Entwicklung von Kirche und Sozialdemokratie betraf. Der ideelle und organisatorische Einfluß der Sozialdemokraten auf die deutsche Arbeiterschaft sowie eine mögliche Annäherung zwischen sozialdemokratischer Ideologie und christlichem Gedankengut standen im Zentrum des weiteren Gesprächs ${ }^{77}$.

71 Siehe unten, S. $191 \mathrm{ff}$.

$72 \mathrm{Vgl}$. Soell, Fritz Erler, S. $858 \mathrm{ff}$.

73 Schauff an Erler, 14. 6. 1964 (IfZ, NL Schauff, Bd. 25).

74 Vgl. die Korrespondenz Schauff-Erler, 1.6.-11. 12. 1964 (IfZ, NL Schauff, Bd. 25). Der Besuch, in dessen Ablauf auch der deutsche Vatikanbotschafter Hilger van Scherpenberg involviert war, hatte auf Wunsch des Papstes strikt informellen Charakter.

75 AsD, NL Fritz Erler, Mappe 153.

76 Peter Nellen war von der CDU zur SPD übergetreten. Er war in Rom ebenfalls in die Vorbereitungen des Besuchs eingeschaltet. Vgl. Soell, Fritz Erler, S. 863.

77 Alexander Kohn-Brandenburg, Aufzeichnung für die Genossen (vertraulich), 17. 3. 1964: Der Verlauf des Besuchs der SPD-Delegation in Rom, 4.-6. 3.1964 (AsD, NL Fritz Erler, Mappe 153); Soell, Fritz Erler, S. 862 ff. 
Am 31. Dezember 1966 schrieb Schauff an Kardinal Bea - seit 1960 Leiter des Sekretariats für die Einheit der Christen und während des Konzils der Ansprechpartner vor allem auch für die Nichtkatholiken -, daß er dem Heiligen Vater berichtet habe, daß bereits bei den Vorverhandlungen zur Großen Koalition „zwischen den beiden entscheidenden Persönlichkeiten [Kurt Georg Kiesinger und Herbert Wehner] vereinbart worden sei, im Rahmen der verfassungsändernden Gesetze auch die Kulturfragen und das Verhältnis zu den Kirchen neu und den heutigen Zeitverhältnissen entsprechend zu regeln. Im Einvernehmen mit dem Bundeskanzler, Herrn Wehner und Herrn Bundesminister Leber, der als aktiver Katholik und Gewerkschaftsführer von besonderer Bedeutung sein wird ..., prüfe ich zur Zeit die Form der Vorbereitungsarbeit auf dem Gebiete. "78

Bea nahm diese Vorschläge sehr positiv auf und begrüßte vor allem eine mögliche Revision des Konkordats ${ }^{79}$. Neben Wehner und Leber stand auf sozialdemokratischer Seite vor allem Fritz Erler diesen Schauffschen Bemühungen positiv gegenüber und war in diesem Zusammenhang selbst schon initiativ geworden ${ }^{80}$. Erler, der sich vergeblich darum bemüht hatte, den Besuch der SPD-Delegation im Vatikan 1964 aus dem "Strudel parteipolitischer Auseinandersetzungen" in Deutschland herauszuhalten ${ }^{81}$, erkannte wohl besonders deutlich, daß die Sozialdemokratie das "philosophisch-aufklärerische Denken“ als Erbe des 19. Jahrhunderts überwinden und „ein neues Bewußtsein von der Rolle“ gewinnen müsse, "die auch die religiösen Bindungen in unserer Zeit spielen"82.

Bei Betrachtung der Haltung der deutschen Bischöfe in der Frage der Annäherung der SPD an die Katholische Kirche argumentierte auch der in Münster amtierende Heinrich Tenhumberg im gleichen Sinne; er stimmte mit Schauff darin überein, daß die „Verkrustungen aus der Kulturkampfzeit" abgeworfen werden müßten. Tenhumbergs Plädoyer für einen Brückenschlag zwischen Sozialdemokratie und Kirche und seine öffentlich bekundete „innere Nähe“ $z u$ Herbert Wehner provozierten jedoch Kritik innerhalb des Episkopats wie auch bei Teilen der katholischen Bevölkerung83. Diese Reaktion löste bei Schauff „böse Erinnerungen an das Freund-Feind-Denken der Dreißiger Jahre “ aus, bestärkte aber zugleich auch den weiteren freundschaftlich-kritischen Dialog mit dem Münsteraner

78 Schreiben Schauff an Kardinal Bea, 31. 12. 1966 (IfZ, NL Schauff, Bd. 25). Annemarie Renger vermutet also richtig, daß der Heilige Stuhl durch Schauff über die Verhandlungen über die Bildung einer Großen Koalition unterrichtet war, doch war dieser kein „Vertreter" des Vatikans (Renger, Leben, S. 211 f.).

79 Schreiben Kardinal Bea an Schauff, 7. 1. 1967. Das Schreiben wurde von Schauff an Wehner und Bischof Tenhumberg weitergeleitet (IfZ, NL Schauff, Bd. 25).

$80 \mathrm{Vgl}$. den Briefwechsel Schauff-Erler in dieser Angelegenheit (AsD, NL Fritz Erler, Mappe 153, sowie IfZ, NL Schauff, Bd. 25).

81 Vgl. Frankfurter Rundschau, 6. März 1964; Soell, Fritz Erler, S. 864 ff.

82 Vgl. das Interwiew mit Pater Paul Schröder SJ in einer Fernsehsendung des Südwestfunks über den Rom-Besuch der SPD-Delegation, 29. 4. 1964 (Soell, Fritz Erler, S. 865f.).

83 Tenhumberg hatte am 19. April 1970 innerhalb der vom ZDF gesendeten Fernsehreihe „Mitra, Macht und Management" zum Verhältnis SPD-Katholische Kirche Stellung genommen. Die Replik auf seine Kritiker in: Publik vom 1. 5. 1970. 
Bischof ${ }^{84}$. In der Bundesrepublik bemühte sich Schauff unterdessen mit Wehner, der ihn dabei unterstützte, die Diskussion über Konkordats- und Schulfragen in Gang zu bringen.

Ein weiteres brisantes Thema war die diplomatische Neuregelung der polnischen Diözesangrenzen als eine der Voraussetzungen zu einem gutnachbarlichen Verhältnis ${ }^{85}$. Schauff schaltete Wehner auch im Zusammenhang mit personalpolitischen Fragen der deutschen Botschaft beim Vatikan ein, um zu verhindern, daß in der Abstimmung der deutschen mit der vatikanischen Ostpolitik dort Diplomaten mit der „Mentalität vieler deutscher Flüchtlingsvertreter" Fuß faßten; Vatikanbotschafter Berger, ein engagierter Gegner der sozialdemokratischen Ostpolitik, wurde bei diesem Versuch personalpolitischer Einflußnahme regelrecht "geschnitten" 86 .

Im November 1969 schließlich - Schauff hatte vorab das vatikanische Staatssekretariat informiert ${ }^{87}$ - oblag es nichtsdestoweniger der deutschen Vertretung beim Heiligen Stuhl, den ersten Besuch von Georg Leber und Herbert Wehner im Vatikan zu arrangieren. Nach Gesprächen mit dem Substituten des Staatssekretariats, Msgr. Benelli, und Bischof Casaroli ${ }^{88}$, an denen auch Botschafter Berger teilnahm, erfolgte eine halbstündige Audienz bei Papst Paul VI. Dabei wurde über die Entspannungsbemühungen der inzwischen installierten sozialliberalen Koalition im allgemeinen gesprochen, im besonderen jedoch über die mögliche Akzeptanz der Oder-Neiße-Grenze und der damit verbundenen Neuregelung der Diözesangrenzen ${ }^{89}$.

Im Sommer des darauffolgenden Jahres reiste Bundeskanzler Brandt selbst an den Tiber und wurde im Vatikan von Papst Paul VI. empfangen. In die Vorbereitung auch dieser Reise war Johannes Schauff eingeschaltet ${ }^{90}$ - ein Engagement, das ihm in kirchlich-konservativen Kreisen der Bundesrepublik nicht nur

84 Korrespondenz Schauff-Tenhumberg, IfZ, NL Schauff, Bd. 8; das betreffende Schreiben datiert vom 4. 5. 1970.

85 Schreiben Schauff an Wehner vom 6. 3. 1967 (IfZ, NL Schauff, Bd. 38).

86 Schauff an Wehner am 3.4. 1969, IfZ, NL Schauff, Bd. 38; KNA-Informationsdienst, Nr. 50, 11. 12. 1959, S. 4.

87 „Inzwischen habe ich auf persönlichen Kanälen das vatikanische Staatssekretariat über den beabsichtigten Besuch von Ihnen und Georg Leber mit einem Kommentar über das Programm der neuen Regierung sowie der vorgesehenen Gesprächspunkte sowie Besuchsabsichten (Justitia et Pax', Kardinal Bea etc.) vertraulich wissen lassen. Nächste Tage bin ich selbst im Staatssekretariat." (Schreiben an Wehner, o. D.); ein entsprechender Vermerk zum „Besuch der Minister Wehner und Leber beim Hl. Stuhl“ mit einem „Vorläufigen Besuchsprogramm“ am 17./18.11. in Rom datiert vom 22. 10. 1969 (IfZ, NL Schauff, Bd. 38 und 39).

88 Agostino Casaroli (ab 1979 Kardinalstaatssekretär) war zu dieser Zeit Sekretär des Rats für die öffentlichen Angelegenheiten der Kirche; seine diplomatischen Aktivitäten erstreckten sich vor allem auf Polen und die übrigen Länder des Ostblocks.

89 IfZ, NL Schauff, Bd. 25; Der Spiegel, Nr. 23/1969, S. 24 und 53/1969, S. 23.

90 Vgl. Schauffs Korrespondenz mit Georg Leber, dem er am 25. 6. 1970 Vorschläge zur Organisation dieses Besuchs und einen Entwurf für die geplante Ansprache des Bundeskanzlers im Campo Santo (Teutonico) unterbreitete, darunter auch die zustimmende Erwähnung einer Regelung der westpolnischen Diözesanfrage („Minister Ehmke will Schauff selbst ins Bild setzen“), IfZ, NL Schauff, Bd. 5. 
Freunde schuf ${ }^{91}$ und auch zum Bruch der bis dahin freundschaftlichen Beziehung zu Botschafter Berger führte ${ }^{92}$. Diese Entfremdung reichte in die Reihen weiterer politischer Freunde aus dem Unionslager. So sah Rainer Barzel in Schauff einen „Mann des Vatikans“, den Wehner gezielt benutzt habe, „um die Fäden zu Heinrich Lübke und Heinrich Krone zu ziehen, auf daß es zur Großen Koalition“ komme ${ }^{93}$.

Schauffs römische Aktivitäten - Beobachter sahen in ihm zu dessen Mißfallen den "Nebenbotschafter" von Berger" ${ }^{94}$ - hatten jedoch noch einen besonderen Aspekt. In Rom war es schon während des Zweiten Vatikanischen Konzils und im Zusammenhang mit der Diskussion der polnischen Frage sowie der Kontaktaufnahme der deutschen Sozialdemokraten mit dem Heiligen Stuhl zur Konkretisierung von Beziehungen gekommen, die Schauff bereits nach seiner Rückkehr aus dem Exil geknüpft hatte - u. a. auch mit General Reinhard Gehlen, dem Chef des damals entstehenden Bundesnachrichtendienstes ${ }^{95}$. Seine Berichterstattung nach Pullach aus Rom diente auf Gehlens Wunsch vor allem auch der Information des damaligen Bundeskanzlers Kiesinger ${ }^{96}$ und war nach der Ablösung Gehlens durch General Gerhard Wessel im Mai 1968 zunächst ausgesetzt worden ${ }^{97}$.

Von der Zweckmäßigkeit dieser spezifischen Schauffschen Berichterstattung war vor allem Theodor von und zu Guttenberg überzeugt, damals Parlamentarischer Staatssekretär im Bundeskanzleramt. Guttenberg wirkte über Staatssekretär Karl Carstens auf Wessel ein, seine Entscheidung hinsichtlich der Einstellung der Schauffschen Berichterstattung zu revidieren. Daraufhin fand ein klärendes $\mathrm{Ge}-$ spräch zwischen dem neuen Präsidenten des BND und Schauff statt ${ }^{98}$, demzufolge seine Arbeit in Rom weitergeführt und sogar "noch erweitert werden" sollte ${ }^{99}$.

91 Vgl. den Briefwechsel Schauff-Morsey, 8. und 22. 12. 1971 (IfZ, NL Schauff, Bd. 5).

92 Vgl. Brief Karin Schauff an Berger vom 23. 10. 1971, in dem sie diesem anläßlich seines Ausscheidens aus dem Amt mitteilt, „wie leid es mir tut, daß ein langes und erprobtes Einvernehmen so stark getrübt werden konnte, daß Sie ohne Abschied gehen wollen“ (ACDP, NL Berger, I-400-036/3).

93 Barzel, Geschichten, S. 91.

$94 \mathrm{Vgl}$. Vermerk Berger über eine Unterhaltung mit Leisler/Springer-Auslandsdienst vom 10.1. 1970 („Ich sagte Leisler, ich würde eine Nebenregierung nicht dulden“), ACDP, NL Berger, I-400-034/3; Schreiben Berger an Schauff vom 22. 4. 1970 in IfZ, NL Schauff, Bd. 1.)

${ }^{95}$ Schauff berichtete dem Verfasser, daß er mit Gehlen vor allem beim Ausbau der Beziehungen der Bundesrepublik zu den USA zusammengearbeitet habe.

96 Vgl. die entprechende Notiz im NL Guttenberg, BA Koblenz, Bd. 42 , Bl. 115.

97 Krone, Tagebücher, 13. 2. 1969: "Jetzt haben sie Hans Schauff den Stuhl vor die Tür gesetzt. Man benötigt seine Dienste nicht mehr, und Globke und ich bekommen an Nachrichten auch nichts mehr. - Der neue Wind in Pullach" (ACDP, NL Krone, I-028-073/5).

98 Am 17. 3. 1969 schieb General Wessel an Guttenberg: „Am 14. März hat mich Herr Dr. Schauff besucht. Wir haben ein mehrstündiges Gespräch geführt, das - wie ich glaube für beide Seiten von Wert und Interesse war. Ich jedenfalls bin dankbar, daß dieses, letztlich wohl durch Ihre Initiative angeregte Treffen mir endlich ein klares Bild vermittelt hat, das mich in die Lage versetzt, die Verbindung mit allem, was dazu gehört, unverändert fortführen zu können." (BA, NL Guttenberg, Bd. 42, Bl. 116).

99 Notiz Krone, Tagebücher, 14. 3. 1969, ACDP, I-028-073/5; im Informationsdienst der 
Schauffs Verbindungen zum BND trugen ihm in der Presse das Etikett "Agent" ein ${ }^{100}$. Die Verbindung zu Pullach, die er ja nie in Abrede stellte, gehörte für ihn allerdings in den Gesamtzusammenhang der antikommunistischen und antitotalitären Politik des Westens und insbesondere der Bundesrepublik. Herbert Wehner hatte Schauffs Verbindungen zum BND gekannt und auch unterstützt ${ }^{101}$. In diesem Umfeld traf Schauff jedoch auch auf weitere politische Weggefährten aus der Vergangenheit, insbesondere aus der Emigration, unter denen der Name von Klaus Dohrn besonders hervorzuheben ist ${ }^{102}$.

KNA vom 18. 3. 1982 wird Schauffs Rolle als Berichterstatter aus Rom an den BND eher beiläufig erwähnt; als Informanten werden zwei Monsignori genannt.

100 Vgl. u. a. Konkret (Konkret-Extra), 3/1982, bes. S. 6; Der Spiegel, 37/1982, S. 30 f. (beide Berichte berufen sich auf ihnen vorliegende nicht veröffentlichte Aufzeichnungen des ehemaligen Staatsschutzbeamten Hans Langemann, der in den siebziger Jahren als BNDResident an der deutschen Botschaft in Rom tätig war).

101 Ein entsprechender Hinweis in Konkret, 3/1982, S. 6.

102 Siehe dazu unten, S. 214. 